PROCEEDINGS OF THE

AMERICAN MATHEMATICAL SOCIETY

Volume 139, Number 10, October 2011, Pages 3707-3717

S 0002-9939(2011)11039-6

Article electronically published on April 1, 2011

\title{
HARNACK INEQUALITY \\ FOR THE NEGATIVE POWER GAUSSIAN CURVATURE FLOW
}

\author{
YI LI
}

(Communicated by Jianguo Cao)

\begin{abstract}
In this paper, we study the power of Gaussian curvature flow of a compact convex hypersurface and establish its Harnack inequality when the power is negative. In the Harnack inequality, we require that the absolute value of the power is strictly positive and strictly less than the inverse of the dimension of the hypersurface.
\end{abstract}

\section{INTRODUCTION}

The Harnack estimate or Harnack inequality plays an important role in geometric flows. For the heat equation, P. Li and S.-T. Yau [6] obtained the corresponding Harnack inequality by using the parabolic maximum principle. Hamilton [4, 5] proved a Harnack inequality for the Ricci flow and mean curvature flow for all dimensions. For a Harnack inequality for $m$-power mean curvature flow, we refer to [1], 9] and [10, where $m$ is positive. B. Chow [3] considered a Harnack inequality for $m$-power Gaussian curvature flow for $m>0$.

In this paper, we consider the negative power Gaussian curvature flow of a compact convex hypersurface $F_{0}: M^{n} \rightarrow \mathbb{R}^{n+1}$,

$$
\frac{\partial}{\partial t} F(x, t)=\frac{1}{K(x, t)^{b}} \cdot \nu(x, t), 0<b<\frac{1}{n} ; F(x, 0)=F_{0}(x), x \in M^{n} .
$$

Here $K$ is the Gaussian curvature and $\nu$ denotes the outward unit normal vector field. Using the similar argument in 3, we obtain a Harnack inequality for the flow (1.1).

Theorem 1.1. Suppose that $F_{0}: M^{n} \rightarrow \mathbb{R}^{n+1}$ is a compact convex hypersurface. If $0<b<\frac{1}{n}$, then

$$
\frac{\partial}{\partial t}\left(\frac{1}{K(x, t)^{b}}\right)+\left|\nabla\left(\frac{1}{K(x, t)^{b}}\right)\right|_{h}^{2}-\frac{n b}{(1-n b) t}\left(\frac{1}{K(x, t)^{b}}\right) \leq 0,
$$

where the notation $|\cdot|_{h}$ is defined in the next section.

When $\frac{1}{n} \leq b \leq 1$, some interesting results have been derived in [7, where the author considered $n=2$.

Received by the editors August 29, 2010.

2010 Mathematics Subject Classification. Primary 53C44, 53C40.

Key words and phrases. Harnack inequality, negative power Gaussian curvature flow.

(C)2011 American Mathematical Society 


\section{Notation and EVolution EQuations}

2.1. Notation. Suppose that $F: M^{n} \rightarrow \mathbb{R}^{n+1}$ is a hypersurface. The second fundamental form is given by

$$
h_{i j}=-\left\langle\frac{\partial^{2} F}{\partial x^{i} \partial x^{j}}, \nu\right\rangle,
$$

where $\langle\cdot, \cdot\rangle$ denotes the standard metric on $\mathbb{R}^{n+1}$. The induced metric

$$
g_{i j}=\left\langle\frac{\partial F}{\partial x^{i}}, \frac{\partial F}{\partial x^{j}}\right\rangle
$$

on $M$ gives us the mean curvature

$$
H=g^{i j} h_{i j}
$$

and the Gaussian curvature

$$
K=\frac{\operatorname{det}\left(h_{i j}\right)}{\operatorname{det}\left(g_{i j}\right)} .
$$

If $\alpha=\left\{\alpha_{i}\right\}$ and $\beta=\left\{\beta_{i}\right\}$ are 1-forms and $s=\left\{s_{i j}\right\}$ is a symmetric positive definite covariant 2 -tensor, we use the short notation

$$
\langle\alpha, \beta\rangle_{s} \equiv\left\langle\alpha_{i}, \beta_{i}\right\rangle_{s}:=s_{i j}^{-1} \alpha_{i} \beta_{j},
$$

where $\left(s_{i j}^{-1}\right)$ is the inverse matrix of $\left(s_{i j}\right)$. Similarly, if $A=\left\{\alpha_{i j k}\right\}$ and $B=\left\{\beta_{p q r}\right\}$ are covariant 3 -tensors, we define

$$
\langle A, B\rangle_{s} \equiv\left\langle A_{i j k}, B_{i j k}\right\rangle_{s}:=s_{i p}^{-1} s_{j q}^{-1} s_{k r}^{-1} A_{i j k} B_{p q r} .
$$

Finally, we define the Laplacian-type operator by

$$
\square:=h_{i j}^{-1} \nabla_{i} \nabla_{j}
$$

Here $\nabla$ denotes the Levi-Civita connection of the induced metric $g$ on $M$.

Let $M^{n}$ be a convex hypersurface in $\mathbb{R}^{n+1}, \alpha=\left\{\alpha_{i}\right\}$ a 1-form on $M^{n}$, and $\phi$ a smooth function on $M$. We have the following identities (see [2] or [3]):

$$
\begin{aligned}
R_{i j k \ell} & =h_{i \ell} h_{j k}-h_{i k} h_{j \ell}, \\
\nabla_{i} h_{j k} & =\nabla_{j} h_{i k}, \\
\left(\nabla_{i} \nabla_{j}-\nabla_{j} \nabla_{i}\right) \alpha_{k} & =-R_{i j k \ell} g^{\ell p} \alpha_{p}, \\
\nabla_{i} \nabla_{j} F & =-h_{i j} \nu \\
\nabla_{i} \nu & =h_{i j} g^{j k} \nabla_{k} F \\
\nabla_{i} \nabla_{j} \nu & =g^{k \ell} \nabla_{k} h_{i j} \cdot \nabla_{\ell} F-g^{\ell k} h_{i \ell} h_{k j} \nu, \\
\nabla_{i}\left(K h_{i j}^{-1}\right) & =0, \\
\left(\nabla_{i} \square-\square \nabla_{i}\right) \phi & =-\left\langle\nabla_{i} h_{j k}, \nabla_{j} \nabla_{k} \phi\right\rangle_{h}-(n-1) h_{i j} g^{j k} \nabla_{k} \phi .
\end{aligned}
$$

2.2. Evolution equations. Now we consider a generalized Gaussian curvature flow

$$
\frac{\partial}{\partial t} F(x, t)=-f(K(x, t)) \cdot \nu(x, t), \quad F(x, 0)=F_{0}(x), x \in M^{n},
$$

where $f:(0,+\infty) \rightarrow \mathbb{R}$ is a smooth function depending only on the Gaussian curvature $K$, which satisfies $f^{\prime}>0$ everywhere in order to guarantee a short time existence. Such a type of Gaussian curvature flow is called the $f$-Gaussian curvature flow. 
Remark 2.1. For convenience, in what follows, we write $f_{t}=f\left(K_{t}\right)$ and $\partial_{2}=\frac{\partial}{\partial_{t}}$.

Under the $f$-Gaussian curvature flow, it is easy to verify the following evolution equations (compared with Lemma 3.1 in [3]), where $h_{t}=\left\{\left(h_{t}\right)_{i j}\right\}$ :

$$
\begin{aligned}
\partial_{t}\left(g_{t}\right)_{i j} & =-2 f_{t}\left(h_{t}\right)_{i j} \\
\partial_{t} \nu_{t} & =\nabla f_{t}=f_{t}^{\prime} \cdot \nabla K_{t}, \\
\partial_{t}\left(h_{t}\right)_{i j} & =\nabla_{i} \nabla_{j} f_{t}-f_{t}\left(g_{t}\right)^{k \ell}\left(h_{t}\right)_{i k}\left(h_{t}\right)_{\ell j}, \\
\partial_{t} K_{t} & =f_{t}^{\prime} K_{t} \cdot\left(\square_{t} K_{t}+\frac{f_{t}^{\prime \prime}}{f_{t}^{\prime}}\left|\nabla K_{t}\right|_{h_{t}}^{2}+\frac{f_{t}}{f_{t}^{\prime} K_{t}} H_{t} K_{t}\right), \\
\partial_{t} f_{t} & =f_{t}^{\prime} K_{t} \cdot\left[\square_{t} f_{t}+H_{t} f_{t}\right], \\
\partial_{t} H_{t} & =\Delta_{t} f_{t}+f_{t}\left|h_{t}\right|_{g_{t}}^{2}, \\
\partial_{t} \square_{t} & =-\left\langle\nabla \nabla f_{t}, \nabla \nabla\right\rangle_{h_{t}}+f_{t} \Delta_{t}+\left(2-n+\frac{f_{t}}{f_{t}^{\prime} K_{t}}\right)\left\langle\Delta_{t} f_{t}, \Delta_{t}\right\rangle_{g}
\end{aligned}
$$

Remark 2.2. If $M^{n}$ is compact and convex, then $H=H_{0}>0$; using the evolution equation (2.20), we see that $H(x, t)=H_{t}(x)>0$ under the $f$-Gaussian curvature flow. According to (2.4), we conclude that $K(x, t)>0$ along the $f$-Gaussian curvature flow. Therefore $\frac{1}{K_{t}}$ is well-defined.

\section{HARNACK INEQUALITY}

Motivated by the self-similar solutions in [3], we define a time-dependent tensor field $P_{t}=\left\{\left(P_{t}\right)_{i j}\right\}$ by

$$
\left(P_{t}\right)_{i j}=\nabla_{i} \nabla_{j} f_{t}-\left(h_{t}\right)_{k \ell}^{-1} \nabla_{k}\left(h_{t}\right)_{i j} \cdot \nabla_{\ell} f_{t}+f_{t}\left(g_{t}\right)^{k \ell}\left(h_{t}\right)_{i k}\left(h_{t}\right)_{\ell j} .
$$

Taking the trace of $\left(P_{t}\right)_{i j}$ with respect to $\left(h_{t}\right)_{i j}$, we set

$$
\mathbf{P}_{t}=\left(h_{t}\right)_{i j}^{-1}\left(P_{t}\right)_{i j}
$$

Since $\nabla K_{t}=K_{t}\left(h_{t}\right)_{p q}^{-1} \nabla\left(h_{t}\right)_{p q}$ by (2.12), we can rewrite $\mathbf{P}_{t}$ as

$$
\mathbf{P}_{t}=\square_{t} f_{t}+f_{t} H_{t}-\left(h_{t}\right)_{i j}^{-1}\left(h_{t}\right)_{k \ell}^{-1} \nabla_{k}\left(h_{t}\right)_{i j} \cdot \nabla_{\ell} f_{t}=\square_{t} f_{t}+f_{t} H_{t}-\frac{\left|\nabla f_{t}\right|_{h_{t}}^{2}}{f_{t}^{\prime} K_{t}}
$$

3.1. Evolution equation for $\mathbf{P}_{t}$. In this subsection our task is to find the evolution equation for $\mathbf{P}_{t}$. Before doing this, we first write down some elementary formulas which will be used in our complicated and tedious computation. Since $f$ is smooth depending only on $K_{t}$, we have $\nabla f_{t}=f_{t}^{\prime} \nabla K_{t}$ and

$$
\begin{aligned}
\square_{t} f_{t} & =\left(h_{t}\right)_{i j}^{-1} \nabla_{i} \nabla_{j} f_{t}=\left(h_{t}\right)_{i j}^{-1} \nabla_{i}\left(f_{t}^{\prime} \nabla_{j} K_{t}\right) \\
& =\left(h_{t}\right)_{i j}^{-1}\left[f_{t}^{\prime \prime} \nabla_{i} K_{t} \cdot \nabla_{j} K_{t}+f_{t}^{\prime} \nabla_{i} \nabla_{j} K_{t}\right]=f_{t}^{\prime} \square_{t} f_{t}+f_{t}^{\prime \prime}\left|\nabla K_{t}\right|_{h_{t}}^{2} .
\end{aligned}
$$

Using $\nabla_{i} K_{t}=\nabla_{i} f_{t} / f_{t}^{\prime}$, we obtain

$$
\nabla_{i} \nabla_{j} K_{t}=\nabla_{i}\left(f_{t}^{\prime-1} \nabla_{i} f_{t}\right)=-f_{t}^{\prime-3} f_{t}^{\prime \prime} \nabla_{i} f_{t} \cdot \nabla_{j} f_{t}+f_{t}^{\prime-1} \nabla_{i} \nabla_{j} f_{t} .
$$


The next useful formula is

$$
\begin{aligned}
&\left.\square_{t}\left(f_{t}^{\prime}\right) K_{t}\right)=\left(h_{t}\right)_{i j}^{-1} \nabla_{i}\left(f_{t}^{\prime \prime} \nabla_{j} K_{t} \cdot K_{t}+f_{t}^{\prime} \cdot \nabla_{j} K_{t}\right) \\
&=\left(h_{t}\right)_{i j}^{-1}\left[f_{t}^{\prime \prime} K_{t} \nabla_{i} \nabla_{j} K_{t}+\left(f_{t}^{\prime \prime \prime} K_{t}+2 f_{t}^{\prime \prime}\right) \nabla_{i} K_{t} \cdot \nabla_{j} K_{t}+f_{t}^{\prime} \nabla_{i} \nabla_{j} K_{t}\right] \\
&=\left(h_{t}\right)_{i j}^{-1}\left[f_{t}^{\prime}+f_{t}^{\prime \prime} K_{t}\right]\left(\frac{1}{f_{t}^{\prime}} \nabla_{i} \nabla_{j} f_{t}-\frac{f_{t}^{\prime \prime}}{f_{t}^{\prime 3}} \nabla_{i} f_{t} \cdot \nabla_{j} f_{t}\right) \\
&+\left(h_{t}\right)_{i j}^{-1}\left[f_{t}^{\prime \prime \prime} K_{t}+2 f_{t}^{\prime \prime}\right] \frac{\nabla_{i} f_{t} \cdot \nabla_{j} f_{t}}{f_{t}^{\prime 2}} \\
&(3.6) \quad\left(1+\frac{f_{t}^{\prime \prime} K_{t}}{f_{t}^{\prime}}\right) \square_{t} f_{t}+\left(\frac{f_{t}^{\prime \prime \prime} K_{t}}{f_{t}^{\prime 2}}+\frac{f_{t}^{\prime \prime}}{f_{t}^{\prime 2}}-\frac{f_{t}^{\prime \prime 2} K_{t}}{f_{t}^{\prime 3}}\right)\left|\nabla f_{t}\right|_{h_{t}}^{2} .
\end{aligned}
$$

Lemma 3.1. Under the f-Gaussian curvature flow, we have

$$
\begin{aligned}
\partial_{t}\left(\square_{t} f_{t}\right)= & f_{t}^{\prime} K_{t} \cdot \square_{t}\left(\square_{t} f_{t}\right)+2\left(1+\frac{f_{t}^{\prime \prime} K_{t}}{f_{t}^{\prime}}\right)\left\langle\nabla f_{t}, \nabla\left(\square_{t} f_{t}\right\rangle_{h_{t}}\right. \\
& +\left(1+\frac{f_{t}^{\prime \prime} K_{t}}{f_{t}^{\prime}}\right)\left(\square_{t} f_{t}\right)^{2}+f_{t}^{\prime} K_{t} \cdot \square_{t}\left(H_{t} f\left(K_{t}\right)\right) \\
& +2\left(1+\frac{f_{t}^{\prime \prime} K_{t}}{f_{t}^{\prime}}\right)\left\langle\nabla f_{t}, \nabla\left(H_{t} f_{t}\right)\right\rangle_{h_{t}}+\left(1+\frac{f_{t}^{\prime \prime} K_{t}}{f_{t}^{\prime}}\right) H_{t} f_{t} \cdot \square_{t} f_{t} \\
& +\left(\frac{f_{t}^{\prime \prime \prime} K_{t}}{f_{t}^{\prime 2}}+\frac{f_{t}^{\prime \prime}}{f_{t}^{\prime 2}}-\frac{f_{t}^{\prime \prime 2} K_{t}}{f_{t}^{\prime 3}}\right)\left|\nabla f_{t}\right|_{h_{t}}^{2}\left[\square_{t} f_{t}+H_{t} f_{t}\right] \\
& -\left|\nabla \nabla f_{t}\right|_{h_{t}}^{2}+f_{t} \Delta_{t} f_{t}+\left(2-n+\frac{f_{t}}{f_{t}^{\prime} K_{t}}\right)\left|\nabla f_{t}\right|_{g_{t}}^{2} .
\end{aligned}
$$

Proof. From $\partial_{t}\left(\square_{t} f_{t}\right)=\left(\partial_{t} \square_{t}\right) f_{t}+\square_{t}\left(\partial_{t} f_{t}\right)$, we get

$\partial_{t}\left(\square_{t} f_{t}\right)=-\left|\nabla \nabla f_{t}\right|_{h_{t}}^{2}+f_{t} \Delta_{t} f_{t}+\left(2-n+\frac{f_{t}}{f_{t}^{\prime} K_{t}}\right)\left|\nabla f_{t}\right|_{g_{t}}^{2}+\square_{t}\left[f_{t}^{\prime} K_{t}\left(\square_{t} f_{t}+H_{t} f_{t}\right)\right]$.

Now we evaluate the last term:

$$
\begin{aligned}
& \square_{t}\left[f_{t}^{\prime} K_{t}\left(\square_{t} f_{t}+H_{t} f_{t}\right)\right]=\square_{t}\left(f_{t}^{\prime} K_{t}\right) \cdot\left(\square_{t} f_{t}+H_{t} f_{t}\right) \\
& \quad+f_{t}^{\prime} K_{t} \cdot \square_{t}\left(\square_{t} f_{t}+H_{t} f_{t}\right)+2\left\langle\nabla\left(f_{t}^{\prime} K_{t}\right), \nabla\left(\square_{t} f_{t}+H_{t} f_{t}\right)\right\rangle_{h_{t}} \\
& =\left(\left(1+\frac{f_{t}^{\prime \prime} K}{f_{t}^{\prime}}\right) \square_{t} f_{t}+\left(\frac{f_{t}^{\prime \prime \prime} K_{t}}{f_{t}^{\prime 2}}+\frac{f_{t}^{\prime \prime}}{f_{t}^{\prime 2}}-\frac{f_{t}^{\prime \prime 2} K_{y}}{f_{t}^{\prime 3}}\right)\left|\nabla f_{t}\right|_{h_{t}}^{2}\right)\left(\square_{t} f_{t}+H_{t} f_{t}\right) \\
& \quad+f_{t}^{\prime} K_{t} \cdot \square_{t}\left(\square_{t} f_{t}\right)+f_{t}^{\prime} K_{t} \cdot \square_{t}\left(H_{t} f_{t}\right) \\
& \quad+2\left\langle\left(1+\frac{f_{t}^{\prime \prime} K_{t}}{f_{t}^{\prime}}\right) \nabla f_{t}, \nabla\left(\square_{t} f_{t}\right)+\nabla\left(H_{t} f_{t}\right)\right\rangle_{h_{t}} .
\end{aligned}
$$

Simplifying the above and plugging into the expression of $\partial_{t}\left(\square_{t} f_{t}\right)$, we obtain the required result.

Lemma 3.2. Under the f-Gaussian curvature flow, we have

$$
\begin{aligned}
\partial_{t}\left(\frac{\left|\nabla f_{t}\right|_{h}^{2}}{f_{t}^{\prime} K_{t}}\right)= & \left(f_{t}^{\prime} K_{t}\right) \square_{t}\left(\frac{\left|\nabla f_{t}\right|_{h_{t}}^{2}}{f_{t}^{\prime} K_{t}}\right)+2\left(1+\frac{f_{t}^{\prime \prime} K_{t}}{f_{t}^{\prime}}\right)\left\langle\nabla f_{t}, \nabla\left(\frac{\left|\nabla f_{t}\right|_{h_{t}}^{2}}{f_{t}^{\prime} K_{t}}\right)\right\rangle_{h_{t}} \\
& -2\left|\nabla \nabla f_{t}\right|_{h_{t}}^{2}+2\left\langle\nabla_{i}\left(h_{t}\right)_{j k}, \nabla_{i} f_{t} \cdot \nabla_{j} \nabla_{k} f_{t}\right\rangle_{h_{t}} \\
& +\left(1+\frac{f_{t}^{\prime \prime} K_{t}}{f_{t}^{\prime}}\right) \frac{2 \square_{t} f_{t}\left|\nabla f_{t}\right|_{g}^{2}}{f_{t}^{\prime} K_{t}}
\end{aligned}
$$




$$
\begin{aligned}
& +\left[1+\left(1+\frac{f_{t}^{\prime \prime} K_{t}}{f_{t}^{\prime}}\right) \frac{f_{t}}{f_{t}^{\prime} K_{t}}\right] H_{t}\left|\nabla f_{t}\right|_{h_{t}}^{2} \\
& -\quad\left\langle\nabla_{i} f \cdot \nabla_{j} h_{k l}, \nabla_{j} f \cdot \nabla_{i} h_{k l}\right\rangle_{h}+\left(\frac{f}{f^{\prime} K}+2-n\right)|\nabla f|_{g}^{2} \\
& +2 f_{t}\left\langle\nabla H_{t}, \nabla f_{t}\right\rangle_{h_{t}}-\left.\left[\frac{f_{t}^{\prime \prime 2}}{f_{t}^{\prime 4}}-\frac{f_{t}^{\prime \prime \prime}}{f_{t}^{\prime 3}}+\frac{1}{\left(f_{t}^{\prime} K_{t}\right)^{2}}\right] \nabla f_{t}\right|_{h_{t}} ^{2} .
\end{aligned}
$$

Proof. The proof is similar to that in $[\underline{3}$. We observe first that

$$
\begin{aligned}
\partial_{t} & \left(\left(f_{t}^{\prime} K_{t}\right)^{-1}\left|\nabla f_{t}\right|_{h_{t}}^{2}\right)=\partial_{t}\left(f_{t}^{\prime-1} K_{t}^{-1}\left(h_{t}\right)_{i j}^{-1} \nabla_{i} f_{t} \nabla_{j} f_{t}\right) \\
= & -\frac{1}{f_{t}^{\prime 2}}\left(\frac{f_{t}^{\prime \prime}}{f_{t}^{\prime} K_{t}}+\frac{1}{K_{t}^{2}}\right) f_{t}^{\prime} K_{t}\left(\square_{t} f_{t}+H_{t} f_{t}\right)\left|\nabla f_{t}\right|_{h_{t}}^{2} \\
& +2\left(f_{t}^{\prime} K_{t}\right)^{-1}\left\langle\nabla\left(f_{t}^{\prime} K_{t}\left(\square_{t} f_{t}+H_{t} f_{t}\right)\right), \nabla f_{t}\right\rangle_{h_{t}} \\
& -\left(f_{t}^{\prime} K_{t}\right)^{-1}\left(h_{t}\right)_{i k}^{-1}\left(h_{t}\right)_{j \ell}^{-1}\left(\nabla_{k} \nabla_{\ell} f_{t}-f_{t} g^{p q} h_{k p} h_{q \ell}\right) \nabla_{i} f_{t} \nabla_{j} f_{t} \\
= & -\frac{1}{f_{t}^{\prime 2}}\left(\frac{f_{t}^{\prime \prime}}{f_{t}^{\prime} K_{t}}+\frac{1}{K_{t}^{2}}\right) f_{t}^{\prime} K_{t}\left(\square_{t} f_{t}+H_{t} f_{t}\right)\left|\nabla f_{t}\right|_{h_{t}}^{2} \\
& -\left(f_{t}^{\prime} K_{t}\right)^{-1}\left\langle\nabla_{i} \nabla_{j} f_{t}, \nabla_{i} f_{t} \nabla_{j} f_{t}\right\rangle_{h_{t}}+\left(f_{t}^{\prime} K_{t}\right)^{-1} f_{t}\left|\nabla f_{t}\right|_{g_{t}}^{2} \\
& +2\left\langle\nabla\left(\square_{t} f_{t}+H_{t} f_{t}\right), \nabla f_{t}\right\rangle_{h_{t}}+2\left(f_{t}^{\prime} K_{t}\right)^{-1}\left\langle\nabla\left(f_{t}^{\prime} K_{t}\right), \nabla f_{t}\right\rangle_{h_{t}}\left(\square_{t} f_{t}+H_{t} f_{t}\right) \\
= & \left(1+\frac{f_{t}^{\prime \prime} K_{t}}{f_{t}^{\prime}}\right) \frac{1}{f_{t}^{\prime} K_{t}}\left(\square_{t} f_{t}+H_{t} f_{t}\right)\left|\nabla f_{t}\right|_{h_{t}}^{2}-\frac{1}{f_{t}^{\prime} K_{t}}\left\langle\nabla_{i} \nabla_{j} f_{t}, \nabla_{i} f_{t} \nabla_{j} f_{t}\right\rangle_{h} \\
& +\frac{f_{t}}{f_{t}^{\prime} K_{t}}\left|\nabla f_{t}\right|_{g_{t}}^{2}+2\left\langle\nabla\left(\square_{t} f_{t}+H_{t} f_{t}\right), \nabla f_{t}\right\rangle_{h_{t}} .
\end{aligned}
$$

On the other hand, we compute the Laplacian of $\left(f_{t}^{\prime} K_{t}\right)^{-1}\left|\nabla f_{t}\right|_{h_{t}}^{2}$ with respect to $\left(h_{t}\right)_{i j}$ :

$$
\begin{aligned}
\square_{t} & \left(\left(f_{t}^{\prime} K_{t}\right)^{-1}\left|\nabla f_{t}\right|_{h_{t}}^{2}\right)=\square_{t}\left(f_{t}^{\prime-1} K_{t}^{-1}\left(h_{t}\right)_{i j}^{-1} \nabla_{i} f_{t} \nabla_{j} f_{t}\right) \\
= & \square_{t}\left(\left(f_{t}^{\prime} K_{t}\right)^{-1}\right)\left|\nabla f_{t}\right|_{h_{t}}^{2}+\left(f_{t}^{\prime} K_{t}\right)^{-1} \cdot \square_{t}\left(h_{t}\right)_{i j}^{-1} \cdot \nabla_{i} f_{t} \nabla_{j} f_{t} \\
& +\left(f_{t}^{\prime} K_{t}\right)^{-1}\left(h_{t}\right)_{i j}^{-1}\left(2 \square_{t}\left(\nabla_{i} f_{t}\right) \cdot \nabla_{j} f_{t}+2\left\langle\nabla_{k} \nabla_{i} f_{t}, \nabla_{k} \nabla_{j} f_{t}\right\rangle_{h_{t}}\right) \\
& +4\left\langle\nabla_{k} h_{i j}^{-1}, \nabla_{k} \nabla_{i} f \cdot \nabla_{j} f\right\rangle_{h}\left(f^{\prime} K\right)^{-1} \\
& +2\left\langle\nabla_{k}\left(f_{t}^{\prime} K_{t}\right)^{-1}, \nabla_{k}\left(h_{t}\right)_{i j}^{-1} \cdot \nabla_{i} f_{t} \nabla_{j} f_{t}\right\rangle_{h_{t}} \\
& +4\left\langle\nabla_{k}\left(f_{t}^{\prime} K_{t}\right)^{-1},\left(h_{t}\right)_{i j}^{-1} \nabla_{k} \nabla_{i} f_{t} \cdot \nabla_{j} f_{t}\right\rangle_{h_{t}} \\
= & \square_{t}\left(\left(f_{t}^{\prime} K_{t}\right)^{-1}\right)\left|\nabla f_{t}\right|_{h_{t}}^{2}+\left(f_{t}^{\prime} K_{t}\right)^{-1} \cdot \square\left(h_{t}\right)_{i j}^{-1} \cdot \nabla_{i} f_{t} \nabla_{j} f_{t} \\
& +2\left(f_{t}^{\prime} K_{t}\right)^{-1}\left\langle\square_{t}\left(\nabla f_{t}\right), \nabla f_{t}\right\rangle_{h_{t}} \\
& +2\left(f_{t}^{\prime} K_{t}\right)^{-1}\left|\nabla_{i} \nabla_{j} f_{t}\right|_{h_{t}}^{2}+2\left\langle\nabla_{k}\left(f_{t}^{\prime} K_{t}\right)^{-1}, \nabla_{k}\left(h_{t}\right)_{i j}^{-1}\right\rangle_{h_{t}} \cdot \nabla_{i} f_{t} \nabla_{j} f_{t} \\
& +4\left\langle\nabla_{k}\left(f_{t}^{\prime} K_{t}\right)^{-1} \nabla_{i} f_{t}, \nabla_{k} \nabla_{i} f_{t}\right\rangle_{h_{t}}-4\left(f_{t}^{\prime} K_{t}\right)^{-1}\left\langle\nabla_{k}\left(h_{t}\right)_{i j}, \nabla_{k} \nabla_{i} f_{t} \cdot \nabla_{j} f_{t}\right\rangle_{h_{t}} .
\end{aligned}
$$

We compute some elementary formulas which will be used later. Note that

$$
\begin{aligned}
& \nabla\left(K_{t}^{-1}\right)=-K_{t}^{-2} \nabla K_{t}=-\frac{\nabla f_{t}}{f_{t}^{\prime} K_{t}^{2}}, \\
& \nabla\left(f_{t}^{\prime-1}\right)=-f_{t}^{\prime-2} \nabla f_{t}^{\prime}=-\frac{f_{t}^{\prime \prime}}{f_{t}^{\prime 3}} \nabla f_{t} .
\end{aligned}
$$


Therefore

$$
\begin{aligned}
& \square_{t}\left(K_{t}^{-1}\right)=\left(h_{t}\right)_{i j}^{-1}\left(2 K_{t}^{-3} \nabla_{i} K_{t} \cdot \nabla_{j} K_{t}-K_{t}^{-2} \nabla_{i} \nabla_{j} K_{t}\right) \\
& \quad=\left(h_{t}\right)_{i j}^{-1}\left[\frac{2}{K_{t}^{3}} \frac{\nabla_{i} f_{t} \cdot \nabla_{j} f_{t}}{f_{2}^{\prime 2}}-\frac{1}{K_{t}^{2}}\left(\frac{\nabla_{i} \nabla_{j} f_{f}}{f_{t}^{\prime}}-\frac{f_{t}^{\prime \prime}}{f_{t}^{\prime 3}} \nabla_{i} f_{t} \nabla_{j} f_{t}\right)\right] \\
& \quad=\frac{2}{f_{t}^{\prime 2} K_{t}^{3}}\left|\nabla f_{t}\right|_{h_{t}}^{2}-\frac{1}{f_{t}^{\prime} K_{t}^{2}} \square_{t} f_{t}+\frac{f_{t}^{\prime \prime}}{f_{t}^{\prime 3} K_{t}^{2}}\left|\nabla f_{t}\right|_{h_{t}}^{2} \\
& \quad=\left(\frac{2}{K_{t}}+\frac{f_{t}^{\prime \prime}}{f_{t}^{\prime}}\right) \frac{1}{\left(f_{t}^{\prime} K_{t}\right)^{2}}\left|\nabla f_{t}\right|_{h_{t}}^{2}-\frac{1}{f_{t}^{\prime} K_{t}^{2}} \square_{t} f_{t}, \\
& \square_{t}\left(f_{t}^{\prime-1}\right)=\left(h_{t}\right)_{i j}^{-1}\left(2 \frac{f_{t}^{\prime \prime 2}}{f_{t}^{\prime 3}} \nabla_{i} K_{t} \nabla_{j} K_{t}-\frac{f_{t}^{\prime \prime \prime}}{f_{t}^{\prime 2}} \nabla_{i} K_{t} \nabla_{j} K_{t}-\frac{f_{t}^{\prime \prime}}{f_{t}^{\prime 2}} \nabla_{i} \nabla_{j} K_{t}\right) \\
& \quad=\left(\frac{3 f_{t}^{\prime \prime 2}}{f_{t}^{\prime 5}}-\frac{f_{t}^{\prime \prime \prime}}{f_{t}^{\prime 4}}\right)\left|\nabla f_{t}\right|_{h_{t}}^{2}-\frac{f_{t}^{\prime \prime}}{f_{t}^{\prime 3}} \square_{t} f_{t}, \\
& \langle\nabla \\
& \quad=\frac{f_{t}^{\prime \prime}}{f_{t}^{\prime 4} K_{t}^{2}}\left|\nabla f_{t}\right|_{h_{t}}^{2} .
\end{aligned}
$$

Using these equations, we arrive at

$$
\begin{aligned}
\square_{t}\left(\left(f_{t}^{\prime} K_{t}\right)^{-1}\right)= & \square_{t}\left(f_{t}^{\prime-1}\right) \cdot K_{t}^{-1}+f_{t}^{\prime-1} \cdot \square_{t}\left(K_{t}^{-1}\right)+2\left\langle\nabla f_{t}^{\prime-1}, \nabla K_{t}^{-1}\right\rangle_{h_{t}} \\
= & \left(\frac{3 f_{t}^{\prime \prime 2}}{K_{t} f_{t}^{\prime 5}}-\frac{f_{t}^{\prime \prime \prime}}{K_{t} f_{t}^{\prime 4}}\right)\left|\nabla f_{t}\right|_{h_{t}}^{2}-\frac{f_{t}^{\prime \prime}}{K_{t} f_{t}^{\prime 3}} \square_{t} f_{t} \\
& +\left(\frac{2}{f_{t}^{\prime 3} K_{t}^{3}}+\frac{f_{t}^{\prime \prime}}{f_{t}^{\prime 4} K_{t}^{2}}\right)\left|\nabla f_{t}\right|_{h_{t}}^{2}-\frac{1}{f_{t}^{\prime 2} K_{t}^{2}} \square_{t} f_{t}+\frac{2 f_{t}^{\prime \prime}}{f_{t}^{\prime 4} K_{t}^{2}}\left|\nabla f_{t}\right|_{h_{t}}^{2} \\
= & -\left(1+\frac{f_{t}^{\prime \prime} K_{t}}{f_{t}^{\prime}}\right) \frac{1}{f_{t}^{\prime 2} K_{t}^{2}} \square_{t} f_{t} \\
& +\left(\frac{3 f_{t}^{\prime \prime 2}}{f_{t}^{\prime 4}}-\frac{f_{t}^{\prime \prime \prime}}{f_{t}^{\prime 3}}+\frac{2}{f_{t}^{\prime 2} K_{t}^{2}}+\frac{3 f_{t}^{\prime \prime}}{f_{t}^{\prime 3} K_{t}}\right) \frac{\left|\nabla f_{t}\right|_{h_{t}}^{2}}{f_{t}^{\prime} K_{t}} .
\end{aligned}
$$

The Laplacian of $\left(h_{t}\right)_{i j}^{-1}$ with respect to $\left(h_{t}\right)_{i j}$ is given by

$$
\begin{aligned}
\square\left(\left(h_{t}\right)_{i j}^{-1}\right)= & \left(h_{t}\right)_{k \ell}^{-1} \nabla_{k}\left(-\left(h_{t}\right)_{i p}^{-1}\left(h_{t}\right)_{j q}^{-1} \nabla_{\ell}\left(h_{t}\right)_{p q}\right)=-\left(h_{t}\right)_{i p}^{-1}\left(h_{t}\right)_{j q}^{-1} \square_{t}\left(h_{t}\right)_{p q} \\
& +\left(h_{t}\right)_{k \ell}^{-1}\left(h_{t}\right)_{i p}^{-1}\left(h_{t}\right)_{j r}^{-1} h_{q s}^{-1} \nabla_{k}\left(h_{t}\right)_{r s} \nabla_{\ell}\left(h_{t}\right)_{p q} \\
& +\left(h_{t}\right)_{k \ell}^{-1}\left(h_{t}\right)_{j q}^{-1}\left(h_{t}\right)_{i r}^{-1}\left(h_{t}\right)_{p s}^{-1} \nabla_{k}\left(h_{t}\right)_{r s} \nabla_{\ell}\left(h_{t}\right)_{p q} .
\end{aligned}
$$

So the second term in the expression of $\square_{t}\left(\left(f_{t}^{\prime} K_{t}\right)^{-1}\left|\nabla f_{t}\right|_{h_{t}}^{2}\right)$ is

$$
\begin{aligned}
\square_{t}\left(\left(h_{t}\right)_{i j}^{-1}\right) \cdot \nabla_{i} f_{t} \nabla_{j} f_{t}= & -\left(h_{t}\right)_{i p}^{-1}\left(h_{t}\right)_{j q}^{-1} \square_{t}\left(h_{t}\right)_{p q} \cdot \nabla_{i} f_{t} \nabla_{j} f_{t} \\
& +2\left(h_{t}\right)_{k \ell}^{-1}\left(h_{t}\right)_{i p}^{-1}\left(h_{t}\right)_{j r}^{-1}\left(h_{t}\right)_{q s}^{-1} \nabla_{k}\left(h_{t}\right)_{r s} \nabla_{\ell}\left(h_{t}\right)_{p q} \cdot \nabla_{i} f_{t} \nabla_{j} f_{t} \\
= & -\left\langle\square_{t}\left(h_{t}\right)_{i j}, \nabla_{i} f_{t} \nabla_{j} f_{t}\right\rangle_{h_{t}} \\
& +2\left\langle\nabla_{i} f_{t} \nabla_{j}\left(h_{t}\right)_{k \ell}, \nabla_{j} f_{t} \cdot \nabla_{i}\left(h_{t}\right)_{k \ell}\right\rangle_{h_{t}} .
\end{aligned}
$$

Combining those identities, we have

$$
\begin{aligned}
& \square_{t}\left(\left(f_{t}^{\prime} K_{t}\right)^{-1}\left|\nabla f_{t}\right|_{h_{t}}^{2}\right)=-\left(1+\frac{f_{t}^{\prime \prime} K_{t}}{f_{t}^{\prime}}\right) \frac{1}{\left(f_{t}^{\prime} K_{t}\right)^{2}} \square_{t} f_{t} \cdot\left|\nabla f_{t}\right|_{h_{t}}^{2} \\
& \quad+\left(\frac{3 f_{t}^{\prime \prime 2}}{f_{t}^{\prime 4}}-\frac{f_{t}^{\prime \prime \prime}}{f_{t}^{\prime 3}}+\frac{2}{f_{t}^{\prime 2} K_{t}^{2}}+\frac{3 f_{t}^{\prime \prime}}{f_{t}^{\prime 3} K_{t}}\right) \frac{\left|\nabla f_{t}\right|_{h_{t}}^{4}}{f_{t}^{\prime} K_{t}}-\left(f_{t}^{\prime} K_{t}\right)^{-1}\left\langle\square_{t}\left(h_{t}\right)_{i j}, \nabla_{i} f_{t} \nabla_{j} f_{t}\right\rangle_{h_{t}}
\end{aligned}
$$




$$
\begin{aligned}
& +2\left(f_{t}^{\prime} K_{t}\right)^{-1}\left\langle\nabla_{i} f_{t} \nabla_{i}\left(h_{t}\right)_{k \ell}, \nabla_{j} f_{t} \cdot \nabla_{i}\left(h_{t}\right)_{k \ell}\right\rangle_{h_{t}} \\
& +2\left(f_{t}^{\prime} K_{t}\right)^{-1}\left\langle\nabla_{i}\left(\square_{t} f_{t}\right)+\left\langle\nabla_{i}\left(h_{t}\right)_{j k}, \nabla_{j} \nabla_{k} f_{t}\right\rangle_{h_{t}}+(n-1)\left(h_{t}\right)_{i j}\left(g_{t}\right)^{j k} \nabla_{k} f_{t}, \nabla_{i} f_{t}\right\rangle_{h} \\
& +2\left(f_{t}^{\prime} K_{t}\right)^{-1}\left|\nabla \nabla f_{t}\right|_{h_{t}}^{2}+2\left\langle\nabla_{k}\left(f_{t}^{\prime} K_{t}\right)^{-1}, \nabla_{k}\left(h_{t}\right)_{i j}^{-1}\right\rangle_{h_{t}} \nabla_{i} f_{t} \cdot \nabla_{j} f_{t} \\
& +4\left\langle\nabla_{k}\left(f_{t}^{\prime} K_{t}\right)^{-1} \nabla_{i} f_{t}, \nabla_{k} \nabla_{i} f_{t}\right\rangle_{h_{t}}-4\left(f_{t}^{\prime} K_{t}\right)^{-1}\left\langle\nabla_{k}\left(h_{t}\right)_{i j}, \nabla_{k} \nabla_{i} f_{t} \cdot \nabla_{j} f_{t}\right\rangle_{h_{t}},
\end{aligned}
$$

and we also have

$$
\begin{aligned}
& \square_{t} \\
& \left(\left(f_{t}^{\prime} K_{t}\right)^{-1}\left|\nabla f_{t}\right|_{h_{t}}^{2}\right)=-\left(1+\frac{f_{t}^{\prime \prime} K_{t}}{f_{t}^{\prime}}\right) \frac{1}{\left(f_{t}^{\prime} K_{t}\right)^{2}} \square_{t} f_{t} \cdot\left|\nabla f_{t}\right|_{h_{t}}^{2} \\
& \quad+\left[\frac{3 f_{t}^{\prime \prime 2}}{f_{t}^{\prime 4}}-\frac{f_{t}^{\prime \prime \prime}}{f_{t}^{\prime 3}}+\frac{2}{\left(f_{t}^{\prime} K_{t}\right)^{2}}+\frac{3 f_{t}^{\prime \prime}}{f_{t}^{\prime 3} K_{t}}\right] \frac{\left|\nabla f_{t}\right|_{h_{t}}^{4}}{f_{t}^{\prime} K_{t}} \\
& \quad-\left(f_{t}^{\prime} K_{t}\right)^{-1}\left\langle\square_{t}\left(h_{t}\right)_{i j}, \nabla_{i} f_{t} \nabla_{j} f_{t}\right\rangle_{h_{t}}+2\left(f_{t}^{\prime} K_{t}\right)^{-1}\left\langle\nabla_{i} f_{t} \nabla_{j}\left(h_{t}\right)_{k l}, \nabla_{j} f_{t} \nabla_{i}\left(h_{t}\right)_{k l}\right\rangle_{h_{t}} \\
& \quad+2(n-1)\left(f_{t}^{\prime} K_{t}\right)^{-1}\left|\nabla f_{t}\right|_{g_{t}}^{2}+2\left(f_{t}^{\prime} K_{t}\right)^{-1}\left\langle\nabla\left(\square_{t} f_{t}\right), \nabla f_{t}\right\rangle_{h_{t}} \\
& \quad+2\left[\frac{f_{t}^{\prime \prime}}{f_{t}^{\prime 3} K_{t}}+\frac{1}{\left(f_{t}^{\prime} K_{t}\right)^{2}}\right]\left\langle\nabla_{i}\left(h_{t}\right)_{j k}, \nabla_{i} f_{t} \nabla_{j} f_{t} \nabla_{k} f_{t}\right\rangle_{h_{t}} \\
& \quad-4\left[\frac{f_{t}^{\prime \prime}}{f_{t}^{\prime 3} K_{t}}+\frac{1}{\left(f_{t}^{\prime} K_{t}\right)^{2}}\right]\left\langle\nabla_{i} \nabla_{j} f_{t}, \nabla_{i} f_{t} \nabla_{j} f_{t}\right\rangle_{h_{t}} \\
& \quad-2\left(f_{t}^{\prime} K_{t}\right)^{-1}\left\langle\nabla_{i}\left(h_{t}\right)_{j k}, \nabla_{i} f_{t} \nabla_{j} \nabla_{k} f_{t}\right\rangle_{h_{t}}+2\left(f_{t}^{\prime} K_{t}\right)^{-1}\left|\nabla \nabla f_{t}\right|_{h_{t}}^{2},
\end{aligned}
$$

where we use the identities

$$
\begin{aligned}
\nabla\left(\left(f_{t}^{\prime} K_{t}\right)^{-1}\right) & =-\left(\frac{f_{t}^{\prime \prime}}{f_{t}^{\prime 3} K_{t}}+\frac{1}{f_{t}^{\prime 2} K_{t}^{2}}\right) \nabla f_{t}, \\
\nabla_{k}\left(h_{t}\right)_{i j}^{-1} & =-\left(h_{t}\right)_{i p}^{-1}\left(h_{t}\right)_{j q}^{-1} \nabla_{k}\left(h_{t}\right)_{p q} .
\end{aligned}
$$

From the above equations we obtain

$$
\begin{aligned}
& \partial_{t}\left(\left(f_{t}^{\prime} K_{t}\right)^{-1}\left|\nabla f_{t}\right|_{h_{t}}^{2}\right)-\left(f_{t}^{\prime} K_{t}\right) \square_{t}\left(\left(f_{t}^{\prime} K_{t}\right)^{-1}\left|\nabla f_{t}\right|_{h_{t}}^{2}\right) \\
&=\left(1+\frac{f_{t}^{\prime \prime} K_{t}}{f_{t}^{\prime}}\right) \frac{1}{f_{t}^{\prime} K_{t}}\left(2 \square_{t} f_{t}+H_{t} f_{t}\right)\left|\nabla f_{t}\right|_{h_{t}}^{2} \\
&+\left[4\left(\frac{f_{t}^{\prime \prime} K_{t}}{f_{t}^{\prime}}+1\right)-1\right] \frac{1}{f_{t}^{\prime} K_{t}}\left\langle\nabla_{i} \nabla_{j} f_{t}, \nabla_{i} f_{t} \cdot \nabla_{j} f_{t}\right\rangle_{h_{t}} \\
&+\left[\frac{f_{t}}{f_{t}^{\prime} K_{t}}-2(n-1)\right]\left|\nabla f_{t}\right|_{g_{t}}^{2}+2\left\langle\nabla\left(H_{t} f_{t}\right), \nabla f_{t}\right\rangle_{h_{t}} \\
&-\left[\frac{3 f_{t}^{\prime \prime 2}}{f_{t}^{\prime 4}}-\frac{f_{t}^{\prime \prime \prime}}{f_{t}^{\prime 3}}+\frac{2}{\left(f_{t}^{\prime} K_{t}\right)^{2}}+\frac{3 f_{t}^{\prime \prime}}{f_{t}^{\prime 3} K_{t}}\right]\left|\nabla f_{t}\right|_{h_{t}}^{4} \\
&+\left\langle\square_{t}\left(h_{t}\right)_{i j}, \nabla_{i} f_{t} \cdot \nabla_{j} f_{t}\right\rangle_{h_{t}}-2\left\langle\nabla_{i} f_{t} \cdot \nabla_{j}\left(h_{t}\right)_{k \ell}, \nabla_{j} f_{t} \cdot \nabla_{i}\left(h_{t}\right)_{k \ell}\right\rangle_{h} \\
&-2\left(\frac{f_{t}^{\prime \prime}}{f_{t}^{\prime 2}}+\frac{1}{f_{t}^{\prime} K_{t}}\right)\left\langle\nabla_{i}\left(h_{t}\right)_{j k}, \nabla_{i} f_{t} \cdot \nabla_{j} f_{t} \cdot \nabla_{k} f_{t}\right\rangle_{h_{t}} \\
&+2\left\langle\nabla_{i}\left(h_{t}\right)_{j k}, \nabla_{i} f_{t} \cdot \nabla_{j} \nabla_{k} f_{t}\right\rangle_{h_{t}}-2\left|\nabla \nabla f_{t}\right|_{h_{t}}^{2} .
\end{aligned}
$$

On the other hand, from [2], we get

$$
\begin{aligned}
\square_{t}\left(h_{t}\right)_{i j}= & \frac{1}{f_{t}^{\prime} K_{t}} \nabla_{i} \nabla_{j} f_{t}-\left(1+\frac{f_{t}^{\prime \prime} K_{t}}{f_{t}^{\prime}}\right) \frac{1}{\left(f_{t}^{\prime} K_{t}\right)^{2}} \nabla_{i} f_{t} \nabla_{j} f_{t} \\
& +\left\langle\nabla_{i}(h)_{k \ell}, \nabla_{j}(h)_{k \ell}\right\rangle_{h_{t}}-H_{t}\left(h_{t}\right)_{i j}+n\left(g_{t}\right)^{k \ell}\left(h_{t}\right)_{i k}\left(h_{t}\right)_{\ell j} .
\end{aligned}
$$


Here we use the identity $\nabla_{i} \nabla_{j} f_{t}=f_{t}^{\prime \prime} \nabla_{i} K_{t} \nabla_{j} K_{t}+f_{t}^{\prime} \nabla_{i} \nabla_{j} K_{t}$. Plugging it into the previous formula, we get

$$
\begin{aligned}
\partial_{t}( & \left.\left(f_{t}^{\prime} K_{t}\right)^{-1}\left|\nabla f_{t}\right|_{h_{t}}^{2}\right)-\left(f_{t}^{\prime} K_{t}\right) \square_{t}\left(\left(f_{t}^{\prime} K_{t}\right)^{-1}\left|\nabla f_{t}\right|_{h_{t}}^{2}\right) \\
= & \left(1+\frac{f_{t}^{\prime \prime} K_{t}}{f_{t}^{\prime}}\right) \frac{2}{f_{t}^{\prime} K_{t}} \square_{t} f_{t} \cdot\left|\nabla f_{t}\right|_{h_{t}}^{2}+4\left(1+\frac{f_{t}^{\prime \prime} K_{t}}{f_{t}^{\prime}}\right) \frac{1}{f_{t}^{\prime} K_{t}}\left\langle\nabla_{i} \nabla_{j} f_{t}, \nabla_{i} f_{t} \nabla_{j} f_{t}\right\rangle_{h_{t}} \\
& +\left[\frac{f_{t}}{f_{t}^{\prime} K_{t}}-2(n-1)+n\right]\left|\nabla f_{t}\right|_{g_{t}}^{2}+\left[1+\left(1+\frac{f_{t}^{\prime \prime} K_{t}}{f_{t}^{\prime}}\right) \frac{f_{t}}{f_{t}^{\prime} K_{t}}\right] H_{t}\left|\nabla f_{t}\right|_{h_{t}}^{2} \\
& +2 f_{t}\left\langle\nabla H_{t}, \nabla f_{t}\right\rangle_{h_{t}}-\left[\frac{3 f_{t}^{\prime \prime 2}}{f_{t}^{\prime 4}}-\frac{f_{t}^{\prime \prime \prime}}{f_{t}^{\prime 3}}+\frac{3}{\left(f_{t}^{\prime} K_{t}\right)^{2}}+\frac{4 f_{t}^{\prime \prime}}{f_{t}^{\prime 3} K_{t}}\right]\left|\nabla f_{t}\right|_{h_{t}}^{4} \\
& -\left\langle\nabla_{i} f_{t} \cdot \nabla_{j}\left(h_{t}\right)_{k \ell}, \nabla_{j}\left(f_{t}\right) \cdot \nabla_{i}\left(h_{t}\right)_{k \ell}\right\rangle_{h_{t}} \\
& -2\left(\frac{f_{t}^{\prime \prime} K_{t}}{f_{t}^{\prime}}+1\right) \frac{1}{f_{t}^{\prime} K_{t}}\left\langle\nabla_{i}\left(h_{t}\right)_{j k}, \nabla_{i} f_{t} \nabla_{j} f_{t} \nabla_{k} f_{t}\right\rangle_{h_{t}} \\
& +2\left\langle\nabla_{i}\left(h_{t}\right)_{j k}, \nabla_{i} f_{t} \nabla_{j} \nabla_{k} f_{t}\right\rangle_{h_{t}}-2\left|\nabla \nabla f_{t}\right|_{h_{t}}^{2} .
\end{aligned}
$$

The final step is to compute $\left\langle\nabla_{i} \nabla_{j} f_{f}, \nabla_{i} f_{t} \nabla_{j} f_{t}\right\rangle_{h_{t}}$. We consider

$$
\begin{aligned}
& \left\langle\nabla f_{t}, \nabla\left(\left(f_{t}^{\prime} K_{t}\right)^{-1}\left|\nabla f_{t}\right|_{h_{t}}^{2}\right)\right\rangle_{h_{t}}=-\left(\frac{f_{t}^{\prime \prime}}{f_{t}^{\prime 3} K_{t}}+\frac{1}{K_{t}^{2} f_{t}^{\prime 2}}\right)\left|\nabla f_{t}\right|_{h_{t}}^{4} \\
& \quad-\frac{1}{f_{t}^{\prime} K_{t}}\left\langle\nabla_{i}\left(h_{t}\right)_{j k}, \nabla_{i} f_{t} \nabla_{j} f_{t} \nabla_{k} f_{t}\right\rangle_{h_{t}}+\frac{2}{f_{t}^{\prime} K_{t}}\left\langle\nabla_{i} \nabla_{j} f_{t}, \nabla_{i} f_{t} \nabla_{j} f_{t}\right\rangle_{h_{t}} .
\end{aligned}
$$

Substituting this formula into the evolution equation, we obtain

$$
\begin{aligned}
\partial_{t}( & \left.\left(f_{t}^{\prime} K_{t}\right)^{-1}\left|\nabla f_{t}\right|_{h_{t}}^{2}\right)-\left(f_{t}^{\prime} K_{t}\right) \square_{t}\left(\left(f_{t}^{\prime} K_{t}\right)^{-1}\left|\nabla f_{t}\right|_{h_{t}}^{2}\right) \\
= & 2\left(1+\frac{f_{t}^{\prime \prime} K_{t}}{f_{t}^{\prime}}\right)\left\langle\nabla f_{t}, \nabla\left(\left(f_{t}^{\prime} K_{t}\right)^{-1}\left|\nabla f_{t}\right|_{h_{t}}^{2}\right)\right\rangle_{h_{t}}-2\left|\nabla \nabla f_{t}\right|_{h_{t}}^{2} \\
& +2\left\langle\nabla_{i}\left(h_{t}\right)_{j k}, \nabla_{i} f_{t} \nabla_{j} \nabla_{k} f_{t}\right\rangle_{h_{t}}+\left(1+\frac{f_{t}^{\prime \prime} K_{t}}{f_{t}^{\prime}}\right) \frac{2}{f_{t}^{\prime} K_{t}} \square_{t} f_{t} \cdot\left|\nabla f_{t}\right|_{h_{t}}^{2} \\
& -\left\langle\nabla_{i} f_{t} \nabla_{j}\left(h_{t}\right)_{k l}, \nabla_{j} f_{t} \nabla_{i}\left(h_{t}\right)_{k l}\right\rangle_{h_{t}}+\left(\frac{f_{t}}{f_{t}^{\prime} K_{t}}+2-n\right)\left|\nabla f_{t}\right|_{g_{t}}^{2} \\
& +\left[1+\left(1+\frac{f_{t}^{\prime \prime} K_{t}}{f_{t}^{\prime}}\right) \frac{f_{t}}{f_{t}^{\prime} K_{t}}\right] H_{t}\left|\nabla f_{t}\right|_{h_{t}}^{2}+2 f_{t}\left\langle\nabla H_{t}, \nabla f_{t}\right\rangle_{h_{t}} \\
& -\left[\frac{3 f_{t}^{\prime \prime 2}}{f_{t}^{\prime 4}}-\frac{f_{t}^{\prime \prime \prime}}{f_{t}^{\prime 3}}+\frac{3}{\left(f_{t}^{\prime} K_{t}\right)^{2}}+\frac{4 f_{t}^{\prime \prime}}{f_{t}^{\prime 3} K}\right. \\
& \left.-2\left(1+\frac{f_{t}^{\prime \prime} K_{t}}{f_{t}^{\prime}}\right)\left(\frac{f_{t}^{\prime \prime}}{f_{t}^{\prime 3} K_{t}}+\frac{1}{\left(f_{t}^{\prime} K_{t}\right)^{2}}\right)\right]\left|\nabla f_{t}\right|_{h_{t}}^{4} .
\end{aligned}
$$

The bracket in the last term equals $\frac{f_{t}^{\prime \prime 2}}{f_{t}^{\prime 4}}-\frac{f_{t}^{\prime \prime \prime}}{f_{t}^{\prime 3}}+\frac{1}{\left(f_{t}^{\prime} K_{t}\right)^{2}}$.

Lemma 3.3. Under the f-Gaussian curvature flow, we have

$$
\partial_{t}\left(f_{t} H_{t}\right)=f_{t}^{\prime} K_{t} \cdot\left(\square_{t} f_{t}+H_{t} f_{t}\right) H_{t}+f_{t}\left(\Delta_{t} f_{t}+f_{t}\left|h_{t}\right|_{g_{t}}^{2}\right) .
$$

Proof. This immediately follows from (2.19) and (2.20). 
Lemma 3.4. Under the f-Gaussian curvature flow, we have

$$
\begin{aligned}
\left|P_{t}\right|_{h_{t}}^{2}= & \left|\nabla \nabla f_{t}\right|_{h_{t}}^{2}+\left\langle\nabla_{i} f_{t} \cdot \nabla_{j}\left(h_{t}\right)_{k l}, \nabla_{j} f_{t} \cdot \nabla_{i}\left(h_{t}\right)_{k \ell}\right\rangle_{h_{t}}+f_{t}^{2}\left|h_{t}^{\bullet}\right|_{g_{t}}^{2} \\
& -2\left\langle\nabla_{i}\left(h_{t}\right)_{j k}, \nabla_{i} f_{t} \cdot \nabla_{j} \nabla_{k} f_{t}\right\rangle_{h_{t}}+2 f_{t} \Delta_{t} f_{t}-2 f_{t}\left\langle\nabla H_{t}, \nabla f_{t}\right\rangle_{h_{t}}, \\
\mathbf{P}_{t}^{2}= & \left(\square_{t} f_{t}\right)^{2}+\frac{\left|\nabla f_{t}\right|_{h_{t}}^{4}}{\left(f_{t}^{\prime} K_{t}\right)^{2}}+f_{t}^{2} H_{t}^{2}-2 \frac{\square_{t} f_{t} \cdot\left|\nabla f_{t}\right|_{h_{t}}^{2}}{f_{t}^{\prime} K_{t}} \\
& +2 H_{t} f_{t} \cdot \square_{t} f_{t}-2 \frac{H_{t} f_{t}\left|\nabla f_{t}\right|_{h_{t}}^{2}}{f_{t}^{\prime} K_{t}} .
\end{aligned}
$$

Proof. By definition, we have

$$
\begin{aligned}
\left|P_{t}\right|_{h_{t}}^{2}= & \left(h_{t}\right)_{i k}^{-1}\left(h_{t}\right)_{j l}^{-1}\left[\nabla_{i} \nabla_{j} f_{t} \cdot \nabla_{k} \nabla_{l} f_{t}-\left(h_{t}\right)_{p q}^{-1} \nabla_{p}\left(h_{t}\right)_{i j} \cdot \nabla_{k} \nabla_{l} f_{t}\right. \\
& -f_{t}\left(g_{t}\right)^{k \ell}\left(h_{t}\right)_{i k}\left(h_{t}\right)_{j \ell} \cdot \nabla_{k} \nabla_{l} f_{t}-\left(h_{t}\right)_{p q}^{-1} \nabla_{p}\left(h_{t}\right)_{k \ell} \nabla_{q} f_{t} \nabla_{i} \nabla_{j} f_{t} \\
& +\left(h_{t}\right)_{p q}^{-1}\left(h_{t}\right)_{r s}^{-1} \nabla_{p}\left(h_{t}\right)_{i j} \cdot \nabla_{r}\left(h_{t}\right)_{k \ell} \nabla_{q} f_{t} \nabla_{s} f_{t} \\
& -f_{t}\left(g_{t}\right)^{k \ell}\left(h_{t}\right)_{i k}\left(h_{t}\right)_{j \ell}\left(h_{t}\right)_{p q}^{-1} \nabla_{p}\left(h_{t}\right)_{k \ell} \nabla_{q} f_{t}+f_{t}\left(g_{t}\right)^{p q}\left(h_{t}\right)_{k p}\left(h_{t}\right)_{\ell q} \nabla_{i} \nabla_{j} f_{t} \\
& \left.-f_{t}\left(g_{t}\right)^{r s}\left(h_{t}\right)_{k r}\left(h_{t}\right)_{\ell s}\left(h_{t}\right)_{p q}^{-1} \nabla_{p}\left(h_{t}\right)_{i j} \nabla_{q} f_{t}+f_{t}^{2}\left(h_{t}\right)_{i j}^{2}\left(h_{t}\right)_{k l}^{2}\right] \\
= & \left|\nabla \nabla f_{t}\right|_{h_{t}}^{2}+\left\langle\nabla_{i} f_{t} \nabla_{j}\left(h_{t}\right)_{k l}, \nabla_{j} f_{t} \nabla_{i}\left(h_{t}\right)_{k l}\right\rangle_{h_{t}}+f_{t}^{2}\left|h_{t}^{\bullet}\right|_{g_{t}}^{2} \\
& -2\left\langle\nabla_{i}\left(h_{t}\right)_{j k}, \nabla_{i} f_{t} \nabla_{j} \nabla_{k} f_{t}\right\rangle_{h_{t}}+2 f_{t} \Delta_{t} f_{t}-2 f_{t}\left\langle\nabla H_{t}, \nabla f_{t}\right\rangle_{h_{t}},
\end{aligned}
$$

where $\left(h_{t}\right)_{i j}^{2}:=\left(h_{t}\right)_{i p}\left(h_{t}\right)_{j q}\left(g_{t}\right)^{p q}$. The second equation is obviously proved by using the first one.

As a direct consequence, we obtain

Theorem 3.5. Under the f-Gaussian curvature flow, we have

$$
\begin{aligned}
\partial_{t} \mathbf{P}_{t}= & f_{t}^{\prime} K_{t} \cdot \square_{t} \mathbf{P}_{t}+2\left(1+\frac{f_{t}^{\prime \prime} K_{t}}{f_{t}^{\prime}}\right)\left\langle\nabla f_{t}, \nabla \mathbf{P}_{t}\right\rangle_{h_{t}}+\left|P_{t}\right|_{h_{t}}^{2}+\left(1+\frac{f_{t}^{\prime \prime} K_{t}}{f_{t}^{\prime}}\right) \mathbf{P}_{t}^{2} \\
& +\left[\left(\frac{f_{t}^{\prime \prime} K_{t}}{f_{t}^{\prime}}\right)^{2}-\frac{f_{t}^{\prime \prime} K_{t}}{f_{t}^{\prime}}-\frac{f_{t}^{\prime \prime \prime} K_{t}^{2}}{f_{t}^{\prime}}\right] \frac{\left|\nabla f_{t}\right|_{h_{t}}^{2}}{f_{t}^{\prime} K_{t}}\left(\frac{\left|\nabla f_{t}\right|_{h_{t}}^{2}}{f_{t}^{\prime} K_{t}}-\square_{t} f_{t}-H_{t} f_{t}\right) \\
& +\left(f_{t}^{\prime} K_{t}-\frac{f_{t} f_{t}^{\prime \prime} K_{t}}{f_{t}^{\prime}}-f_{t}\right)\left(H_{t}^{2} f_{t}+H_{t} \cdot \square_{t} f_{t}\right) \\
& +\left[\left(1+\frac{f_{t}^{\prime \prime} K_{t}}{f_{t}^{\prime}}\right) \frac{f_{t}}{f_{t}^{\prime} K_{t}}-1\right] H_{t}\left|\nabla f_{t}\right|_{h_{t}}^{2} .
\end{aligned}
$$

3.2. Harnack inequality for the negative power Gaussian curvature flow.

For the sake of studying, we define three functions for $x>0$,

$$
\begin{aligned}
& \alpha(x)=\left(\frac{x f^{\prime \prime}(x)}{f^{\prime}(x)}\right)^{2}-\frac{x f^{\prime \prime}(x)}{f^{\prime}(x)}-\frac{x^{2} f^{\prime \prime \prime}(x)}{f^{\prime}(x)}, \\
& \beta(x)=x f^{\prime}(x)-\frac{x f(x) f^{\prime \prime}(x)}{f^{\prime}(x)}-f(x), \\
& \gamma(x)=\left(1+\frac{x f^{\prime \prime}(x)}{f^{\prime}(x)}\right) \frac{f(x)}{x f^{\prime}(x)}-1 .
\end{aligned}
$$


Using this simple notation, we can rewrite the evolution equation for $\mathbf{P}_{t}$ as

$$
\begin{aligned}
\partial_{t} \mathbf{P}_{t}= & f_{t}^{\prime} K_{t} \cdot \square_{t} \mathbf{P}_{t}+2\left(1+\frac{f_{t}^{\prime \prime} K_{t}}{f_{t}^{\prime}}\right)\left\langle\nabla f_{f}, \nabla \mathbf{P}_{t}\right\rangle_{h_{t}}+\left|P_{t}\right|_{h_{t}}^{2}+\left(1+\frac{f_{t}^{\prime \prime} K_{t}}{f_{t}^{\prime}}\right) \mathbf{P}_{t}^{2} \\
& +\alpha\left(K_{t}\right) \frac{\left|\nabla f_{t}\right|_{h_{t}}^{2}}{f_{t}^{\prime} K_{t}}\left(\frac{\left|\nabla f_{t}\right|_{h_{t}}^{2}}{f_{t}^{\prime} K_{t}}-\square_{t} f_{t}-H_{t} f_{t}\right) \\
& +\beta\left(K_{t}\right)\left[H_{t}^{2} f_{t}+H_{t} \cdot \square_{t} f_{t}\right]+\gamma\left(K_{t}\right) H_{t}\left|\nabla f_{t}\right|_{h_{t}}^{2} \\
= & f_{t}^{\prime} K_{t} \cdot \square_{t} \mathbf{P}_{t}+2\left(1+\frac{f_{t}^{\prime \prime} K_{t}}{f_{t}^{\prime}}\right)\left\langle\nabla f_{t}, \nabla \mathbf{P}_{t}\right\rangle_{h_{t}}+\left|P_{t}\right|_{h_{t}}^{2}+\left(1+\frac{f_{t}^{\prime \prime} K_{t}}{f_{t}^{\prime}}\right) \mathbf{P}_{t}^{2} \\
& +\left[H_{t} \beta\left(K_{t}\right)-\alpha\left(K_{t}\right) \frac{\left|\nabla f_{t}\right|_{h_{t}}^{2}}{f_{t}^{\prime} K_{t}}\right] \mathbf{P}_{t}+\left[\frac{\beta\left(K_{t}\right)}{f_{t}^{\prime} K_{t}}+\gamma\left(K_{t}\right)\right] H_{t}\left|\nabla f_{t}\right|_{h_{t}}^{2} .
\end{aligned}
$$

Observing that $\gamma(x)=-\beta(x) / x f(x)^{\prime}$ and $\beta^{\prime}(x)=f(x) \alpha(x) / x$, we have

$$
\begin{aligned}
\partial_{t} \mathbf{P}_{t}= & f_{t}^{\prime} K_{t} \square_{t} \mathbf{P}_{t}+2\left(1+\frac{f_{t}^{\prime \prime} K_{t}}{f_{t}^{\prime}}\right)\left\langle\nabla f_{t}, \nabla \mathbf{P}_{t}\right\rangle_{h_{t}}+\left|P_{t}\right|_{h_{t}}^{2}+\left(1+\frac{f_{t}^{\prime \prime} K_{t}}{f_{t}^{\prime}}\right) \mathbf{P}_{t}^{2} \\
& +\left(H_{t} \beta_{t}-\frac{\beta_{t}^{\prime}}{f_{t} f_{t}^{\prime}}\left|\nabla f_{t}\right|_{h_{t}}^{2}\right) \mathbf{P}_{t},
\end{aligned}
$$

where $\beta_{t}=\beta\left(K_{t}\right)$.

To obtain the Harnack inequality for the negative power Gaussian curvature flow, we should impose some natural condition on $f$. First we investigate some properties of the above three functions associated to the function $f$.

Lemma 3.6. We have

(a) $\alpha \equiv 0$ if and only if $f^{\prime}(x)=a x^{b}$ for some $a>0$ and $b \in \mathbb{R}$;

(b) $\beta \equiv 0$ if and only if $f(x)=a x^{b}$ for $a b>0$.

Proof. Suppose $\alpha \equiv 0$. Then $x\left(f^{\prime \prime 2}-f^{\prime} f^{\prime \prime \prime}\right)=f^{\prime \prime} f^{\prime}$ and hence

$$
x\left(\frac{f^{\prime \prime 2}-f^{\prime} f^{\prime \prime}}{f^{\prime 2}}\right)=f^{\prime \prime} f^{\prime}
$$

which implies that $-x\left(f^{\prime \prime} / f^{\prime}\right)^{\prime}=f^{\prime \prime} / f^{\prime}$. Let $g:=f^{\prime \prime} / f^{\prime}$; so $x g^{\prime}=-g$. Solving this ODE, we get $g=b / x$ for some constant $b$. For (b), putting $g=f^{\prime} / f$ we get $f=a x^{b}$.

When $f(x)=x^{b}$ for $b>0$, B. Chow [3] derived the Harnack inequality for the $f$-Gaussian curvature flow. For the case $b<0$, we give the following:

Theorem 3.7. If $f(x)=a x^{b}$ satisfies (1) $a>0$ and $b>0$, or (2) $a<0$ and $-\frac{1}{n}<b<0$, then

$$
\mathbf{P}_{t} \geq-\frac{1}{\left(\frac{1}{n}+b\right) t}
$$

Consequently,

$$
\frac{\partial f\left(K_{t}\right)}{\partial t}-\left|\nabla f\left(K_{t}\right)\right|_{h}^{2}+\frac{f^{\prime}\left(K_{t}\right) K_{t}}{\left(\frac{1}{n}+b\right) t} \geq 0
$$


Proof. From the above lemma, we have

$$
\partial_{t} \mathbf{P}_{t}=a b K_{t}^{b} \cdot \square_{t} \mathbf{P}_{t}+2 b\left\langle\nabla f_{t}, \nabla \mathbf{P}_{t}\right\rangle_{h_{t}}+\left|P_{t}\right|_{h_{t}}^{2}+b \mathbf{P}_{t}^{2} .
$$

Since $\left|P_{t}\right|_{h_{t}}^{2} \geq \frac{\mathbf{P}_{t}^{2}}{n}$, it follows that

$$
\partial_{t} \mathbf{P}_{t} \geq a b K_{t}^{b} \cdot \square_{t} \mathbf{P}_{t}+2 b\left\langle\nabla f,{ }_{f} \nabla \mathbf{P}_{t}\right\rangle_{h_{t}}+\left(\frac{1}{n}+b\right) \mathbf{P}_{t}^{2} .
$$

The parabolic maximum principle tells us that

$$
\mathbf{P}_{t} \geq-\frac{1}{\left(\frac{1}{n}+b\right) t}
$$

The last inequality is followed by $\partial_{t} f_{t}=f_{t}^{\prime} K_{t}\left(\square_{t} f_{t}+f_{t} H_{t}\right)$.

Now, Theorem 1.1 follows from the above theorem.

\section{REFERENCES}

1. B. Andrews, Harnack inequalities for evolving hypersurfaces, Math. Z., 217(1994), no. 2, 179-197. MR 1296393 (95j:58178)

2. B. Chow, Deforming convex hypersurfaces by the $m$-th root of the Gaussian curvature, J. Differ. Geom., 23(1985), 117-138. MR826427 (87f:58155)

3. B. Chow, On Harnack's inequality and entropy for the Gaussian curvature flow, Comm. Pure. Appl. Math., 44(1991), 469-483. MR1100812 (93e:58032)

4. R. Hamilton, The Harnack estimate for the Ricci flow, J. Differ. Geom., 37(1993), 225-243. MR 1198607 (93k:58052)

5. R. Hamilton, The Harnack estimate for the mean curvature flow, J. Differ. Geom., 41(1995), 215-226. MR:1316556 (95m:53055)

6. P. Li and S.-T. Yau, On the parabolic kernel of the Schrödinger operators, Acta. Math., 156(1986), 153-201. MR834612 (87f:58156)

7. R. Li, Surfaces expanding by the power of the Gauss curvature flow, Proc. Amer. Math. Soc., 138(2010), no. 11, 4089-4102. MR2679630

8. O. Schnürer, Surface expanding by the inverse Gauss curvature flow, J. Reine Angew. Math., 600(2006), 117-134. MR2283800 (2007j:53074)

9. K. Smoczyk, Harnack inequalities for curvature flows depending on mean curvature, New York J. Math., 3(1997), 103-118. MR1480081 (98i:53056)

10. J. Wang, Harnack estimate for $H^{k}$-flow, Science in China Series A: Mathematics, 50(2007), no. 11, 1642-1650. MR2390477 (2008m:53166)

Department of Mathematics, Harvard University, Cambridge, Massachusetts 02138

E-mail address: yili@math.harvard.edu 